\title{
Maturation of Neuromodulatory Effect of Substance P in Rabbit Airways
}

\author{
David T. Tanaka* and Michael M. Grunstein* \\ *Department of Pediatrics, Duke University Medical Center, Durham, North Carolina 27710; and ${ }^{*} J o s e p h$ Stokes, Jr., \\ Research Institute, Children's Hospital of Philadelphia, University of Pennsylvania, Philadelphia, Pennsylvania 19104 ${ }^{1}$
}

\begin{abstract}
The maturation of the neuromodulatory action of substance $\mathbf{P}$ (SP) was investigated in tracheal smooth muscle (TSM) segments isolated from rabbits aged 2-24 wk. The tissues were placed in baths containing Krebs-Ringer solution and contracted with electrical field stimulation (ES) with ES frequencies ranging from 1 to $75 \mathrm{~Hz}$. In tissues $>1$ mo of age, the ES frequency-response relationships were progressively shifted in the presence of a maximally effective neuromodulatory SP dose $\left(10^{-7} \mathrm{M}\right)$ such that by 24 wk of age the mean ( \pm SEM) maximal tension $\left(T_{\max }\right)$ significantly increased from 380.4 ( \pm 41.9$)$ to $502.3( \pm 64.2) \mathrm{g} / \mathrm{g}$ TSM, and the corresponding mean $( \pm \mathrm{SEM}) \log \mathrm{ES}$ frequency producing $50 \%$ of $T_{\max }(\log$ $\left.\mathbf{E S}_{s_{0}}\right)$ significantly decreased from $1.209( \pm 0.069)$ to 1.055 $( \pm 0.046) \mathrm{Hz}$. By contrast, relative to methacholine, the direct contractile effects of SP did not significantly vary with age. In further analyzing the basis for the above age-related difference in the neuromodulatory action of SP, we found that the magnitude of SP-induced neuromodulation was highly correlated to the tissue's intrinsic sensitivity to ES. Indeed, after accounting for the tissue's sensitivity to ES, the effect of age alone on the magnitude of SP-induced neuromodulation was not statistically significant. These findings provide new evidence that: $(a)$ SP-induced neuromodulation of acetylcholine release at the airway neuromuscular junction is significantly enhanced during postnatal development; and (b) that the latter age-dependent action of SP is based on a close coupling of the magnitude of SP-induced neuromodulation to the tissue's intrinsic sensitivity to neurally mediated contraction. (J. Clin. Invest. 1990. 85:345-350.) acetylcholine • development • hyperreactivity • neurokinin • trachea
\end{abstract}

\section{Introduction}

The neurokinin substance $P(S P)^{2}$ is a potent bronchoconstrictor that has been identified in the vagus nerves (1) as well as in nerve fibers supplying the airways in both adult and

This work was presented in abstract form to the Society for Pediatric Research, Washington, DC, 1986.

Address correspondence to Dr. David T. Tanaka, Duke University Medical Center, Box 3179, Durham, NC 27710

Received for publication 13 March 1989 and in revised form 25 September 1989.

1. All experimental work was conducted at the National Jewish Center for Immunology and Respiratory Medicine, Denver, Colorado 80206. 2. Abbreviations used in this paper: ACh, acetylcholine; ES, electrical field stimulation; SP, substance $\mathrm{P}$; $T_{\max }$, maximal isometric tension; TSM, tracheal smooth muscle; TXX, tetrodotoxin.

J. Clin. Invest.

(c) The American Society for Clinical Investigation, Inc.

$0021-9738 / 90 / 02 / 0345 / 06 \$ 2.00$

Volume 85, February 1990, 345-350 immature animals $(2,3)$. In considering its bronchoactive properties, there exists compelling evidence that SP, in addition to its direct effect on airway smooth muscle, may also modulate the release of acetylcholine ( $\mathrm{ACh}$ ) from cholinergic nerve terminals supplying the airways $(4,5)$. Indeed, the contribution of this latter cholinergic component has been identified in separate studies demonstrating a reduction in the magnitude of SP-induced bronchoconstriction after atropine infusion (6), and potentiation by SP administration of neurally mediated airway smooth muscle contraction in vitro $(4,7)$. These findings, together with recent evidence that the atropine-sensitive component of the in vitro bronchoconstrictor responsiveness to SP varies during early postnatal development in the rabbit (6), raise the consideration that the above neuromodulatory effect of SP may be age dependent. In view of the latter, the present study was designed to elucidate the ontogenetic effect of SP on neurally mediated contractility of the isolated airway smooth muscle in maturing rabbits. Our findings herein provide evidence that: $(a)$ the airway neuromodulatory effect of SP is significantly enhanced during postnatal development; and $(b)$ the latter age-related change in the action of SP is coupled to the underlying intrinsic sensitivity of the airway tissue to neurally mediated contraction.

\section{Methods}

Tracheal smooth muscle (TSM) segments $\sim 1 \mathrm{~cm}$ in length were isolated from New Zealand White rabbits of varying age after pentobarbitol anesthesia ( $35 \mathrm{mg} / \mathrm{kg}$ body $\mathrm{wt}$ ) and killing by the injection of an air embolus into a systemic vein. The airway segments were cleaned of loose connective tissue, placed in a siliconized 14- or 20-ml organ bath (Harvard Apparatus Co., Inc., S. Natick, MA), and supported longitudinally by firm stainless steel wire triangular supports. Care was taken to place the membranous portion of the tracheal segment between the wire supports to maximize the recorded tension produced by the isometrically contracting trachealis muscle. The lower support was attached to a glass hook at the base of the organ bath, and the upper support was attached with a gold chain to an isometric force transducer (model FT.03C; Grass Instrument Co., Quincy, MA). The latter was mounted on a rack and pinion clamp so that the resting length of each TSM segment could be adjusted. The tissues were bathed in modified Krebs-Ringer solution (in $\mathrm{mM}$ ) $125 \mathrm{NaCl}, 14 \mathrm{NaHCO}_{3}, 2.25 \mathrm{CaCl}_{2}$ : $2 \mathrm{H}_{2} \mathrm{O}, 1.46 \mathrm{MgSO}_{4}: 7 \mathrm{H}_{2} \mathrm{O}, 4 \mathrm{KCl}, 1.2 \mathrm{NaH}_{2} \mathrm{PO}_{4}: \mathrm{H}_{2} \mathrm{O}$, and $4 \mathrm{~g} /$ liter dextrose. The baths were aerated with a $95 \% \mathrm{O}_{2}$ and $5 \% \mathrm{CO}_{2}$ gas mixture, and a pH of $7.43 \pm 0.03$ was established for the duration of each experiment. The temperatures of the baths were maintained at $37^{\circ} \mathrm{C}$.

Each TSM segment was equilibrated in the bath for $1 \mathrm{~h}$, during which time it was passively stretched on several occasions to a tension of $6 \mathrm{~g}$, and the bath rinsed with fresh buffer solution. The optimal resting length for each tissue was determined by assessing its maximal contractile response to the following standard ES: $8 \mathrm{~V}, 2$-ms pulse duration, $50-\mathrm{Hz}$ stimulation frequency, and $24.5 \mathrm{~mA}$. The ES was delivered by a S48D stimulator (Grass Instrument Co.), connected to a patient stimulus isolation unit (SIU 5; Grass Instrument Co.), and applied transversely across the tissues by means of parallel platinum plate electrodes, each $0.3 \mathrm{~cm}^{2}$. After establishing optimal resting length, the tissues were subjected to varying ES, and noncumulative 
stimulus frequency-response curves were generated by increasing the stimulus frequency from 1 to $75 \mathrm{~Hz}$. Each stimulus frequency was maintained until a peak contractile response was attained, and thereafter the stimulation was terminated to permit $\sim 2$ min of recovery time to elapse between each successive level of stimulation. To assess the effects of SP on neurally mediated contraction of TSM, the responses to ES were re-evaluated after the administration of $10^{-7} \mathrm{M}$ SP (Peninsula Laboratories, Inc., Belmont, CA). Moreover, in both 24- and 2-wk-old rabbits, ES frequency-response curves were also separately determined before and after the administration of $10^{-5} \mathrm{M}$ of the SP antagonist, D-Arg ${ }^{1}$, D-Pro ${ }^{2}$, D-Trp ${ }^{7,9}$, Leu ${ }^{11}$-SP (Peninsula Laboratories, Inc.).

To further evaluate the characteristics of ES-induced TSM contraction, the following agents were separately examined: $(a)$ tetrodotoxin (TTX; $4 \times 10^{-6} \mathrm{M}$ ), an inhibitor of neural transmission (Sigma Chemical Co., St. Louis, MO); (b) atropine sulfate $\left(10^{-6} \mathrm{M}\right)$, a muscarinic cholinergic antagonist (Sigma Chemical Co.); and (c) the cholinergic agonist, acetyl- $\beta$-methacholine $\left(10^{-8}\right.$ to $10^{-4} \mathrm{M}$ ) (Sigma Chemical Co.). All drug concentrations were expressed as final bath concentrations.

At the end of each experiment, the tracheal segments were blotted on gauze pads and weighed. The TSM wet weight was then determined by carefully dissecting free the entire membranous portion of the TSM segment, and then subtracting the remaining airway segment weight from that of the originally intact airway segment. All tensions were normalized for TSM weight, and expressed as grams isometric tension per gram TSM weight. To characterize the frequency-response curves, we determined the maximal isometric tension $\left(T_{\max }\right)$ generated by each TSM segment, as well as the isometric tensions developed at ES frequencies producing 25 and $50 \%$ of $T_{\max }$ (i.e., $\mathrm{ES}_{25}$ and $\mathrm{ES}_{50}$, respectively). The individual determinations of $\mathrm{ES}_{50}$ were converted to their corresponding log values from which the arithmetic means ( \pm SEM) of the $\log \mathrm{ES}_{50}$ values were calculated and used for statistical comparisons of the data. Where appropriate, statistical analyses were performed using either the two-tailed $t$ test or analysis of variance using the SAS general linear models procedure (SAS Procedure GLM; SAS Institute, Cary, NC), and a $P$ value of $<0.05$ was considered significant.

\section{Results}

Age-related effects of SP on ES-induced tracheal contraction. SP administration affected the TSM contractile response to ES in an age-dependent manner, as exemplified by the records obtained in the airway segments isolated from representative 24- and 1-wk-old rabbits in Fig. 1. Here, the same electrical stimulus $(5 \mathrm{~Hz})$ was repeatedly applied to both tissues, with each stimulation lasting $\sim 1 \mathrm{~min}$. The 24-wk-old TSM segment in Fig. $1 A$ demonstrated reproducible contractions on three separate occasions of initial (i.e., control) stimulation, with each contractile response generating $\sim 2 \mathrm{~g}$ of active tension. Subsequently, the administration of SP $\left(10^{-7} \mathrm{M}\right)$ was associated with a small transient TSM contraction that spontaneously resolved after $\sim 1 \mathrm{~min}$, and the same original resting baseline tension was re-established. Thereafter (Fig. $1 B$ ) it can be seen that the magnitude of the ES-induced contractions were increased to $6 \mathrm{~g}$ of active tension. This augmented contractile response to ES was noted to persist for up to $2 \mathrm{~h}$ after SP administration; however, after rinsing the organ bath free of SP with fresh buffer (Fig. $1 C$ ), the TSM response to ES reverted to the initial control level obtained before SP administration. By contrast, the same experiment conducted in the 1-wk-old tissue failed to demonstrate any SP-induced modulation of the neurally mediated contractions. Indeed, as shown in Fig. $1 E$, after SP administration the TSM contractions elicited during ES were similar in magnitude to those obtained both under initial control conditions (Fig. $1 D$ ) and after subsequent rinsing of the organ bath (Fig. $1 F$ ). Moreover, it should be noted that while maximal potentiation of the ES-induced contractions in older tissues was achieved with an SP dose of $10^{-7} \mathrm{M}$ (7), in separate experiments conducted on tissues $\sim 1$ wk old, the ES-induced contractions remained virtually unaffected despite the administration of SP in doses of up to $10^{-4} \mathrm{M}$.

The pooled data from the groups of 11- and 1-wk-old tissues are illustrated in Fig. 2, where for each tissue the isometric tensions obtained at different stimulation frequencies are normalized to the corresponding control $T_{\max }$ generated before SP administration. In all cases, the frequency-response curves obtained in the presence of SP $\left(10^{-7} \mathrm{M}\right)$ were generated after complete recovery of the initial contractile response to the neuropeptide (i.e., see Fig. 1). For the 11-wk-old airway segments (Fig. $2 A$ ) it can be seen that, relative to the control relationship, the mean frequency-response curve obtained in
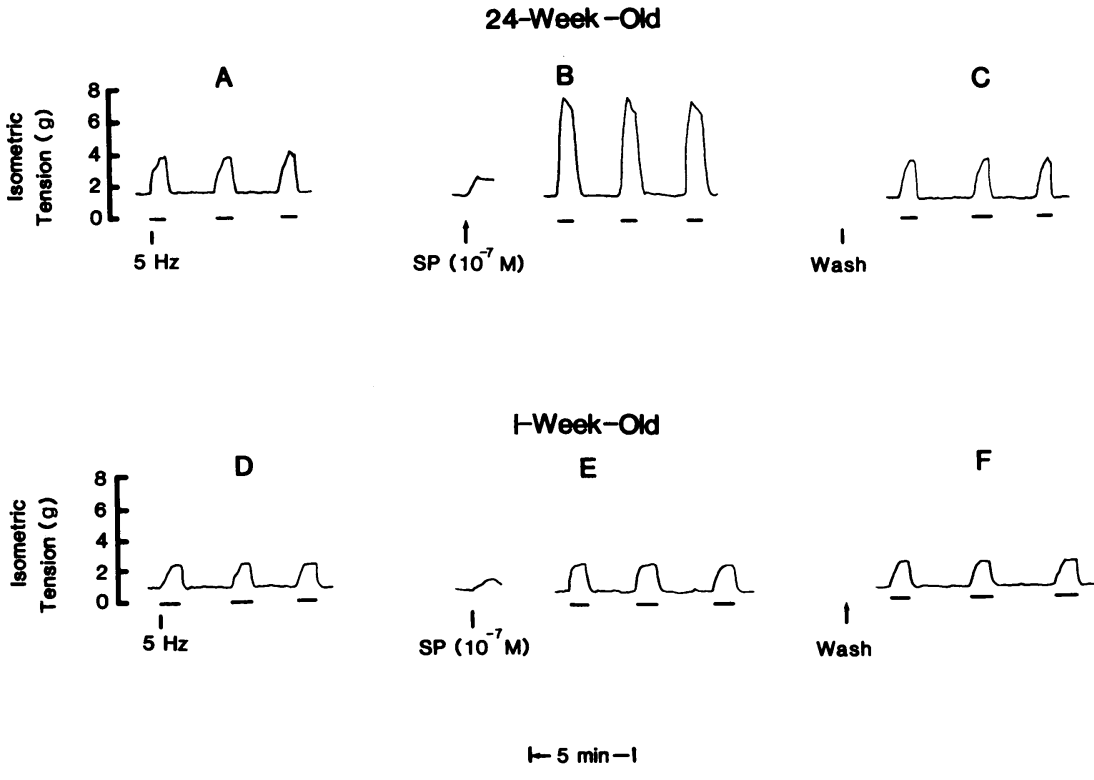

Figure 1. Representative TSM contractile responses to a given ES of $5 \mathrm{~Hz}$ before and after SP administration $\left(10^{-7} \mathrm{M}\right)$ in a 24 -wk-old airway (top tracings) and 1-wk-old airway (bottom tracings). Horizontal bars below tracings indicate duration of ES. $A$ and $D$, control responses to ES before $\mathrm{SP} ; B$ and $E$, ES responses after SP administration; $C$ and $F$, ES responses after washout of SP from organ bath. Note that gaps in tracings in $B$ and $E$ represent $\sim 10 \mathrm{~min}$ required for full resolution of the initial end-organ contractile response to SP. 

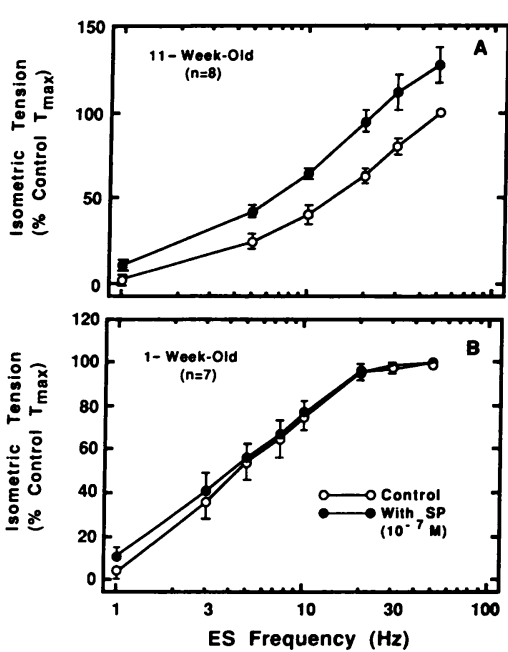

Figure 2. Effect of SP $\left(10^{-7} \mathrm{M}\right)$ on noncumulative ES frequency response relationships obtained in 11-wk-old $(A)$ and 1-wk-old $(B)$ TSM segments. Isometric tensions before (open circles) and in the presence of SP (solid circles) are normalized to the control peak tension $\left(T_{\max }\right)$ responses to $\mathrm{ES}$. Bars, Mean \pm SEM.

the presence of SP was shifted upward, as evidenced by increases in the isometric tensions generated at all frequencies of electrical field stimulation. Consecutive frequency-response curves obtained in untreated tissues were highly reproducible (data not shown). The mean ( \pm SEM) $T_{\max }$ value for tissues treated with SP amounted to $502.3( \pm 64.2) \mathrm{g} / \mathrm{g}$ TSM, which was significantly greater $(P<0.05)$ than the corresponding mean control $T_{\max }$ of $380.4( \pm 41.9) \mathrm{g} / \mathrm{g}$ TSM. In addition, the mean $( \pm \mathrm{SEM}) \log$ stimulus frequency producing $50 \%$ of $T_{\max }$ (i.e., $\left.\log \mathrm{ES}_{50}\right)$ also significantly decreased from $1.206( \pm 0.069)$ $\mathrm{Hz}$ to $1.055( \pm 0.046) \mathrm{Hz}$ after SP administration $(P<0.01)$, indicating an increase in airway sensitivity to ES in the presence of SP. By contrast, in the 1-wk-old tissues (Fig. 2 B), SP administration did not significantly affect the contractile responses to ES generated over the entire range of electrical field stimulation.

Effect of age on SP-induced tracheal contraction. In light of the above age-related differences in the neuromodulatory effect of SP, we further examined whether the intrinsic airway smooth muscle contractile response to SP is also age dependent. Accordingly, we evaluated the dose-dependent effect of SP administration on the end-organ contractile response in TSM segments isolated from 2- and 24-wk-old rabbits. Fig. 3 illustrates the cumulative dose-response curves to SP. The mean $( \pm \mathrm{SEM}) T_{\max }$ values for methacholine in the 2- and 24-wk-old airway segments amounted to $1,765 \pm 252$ and $1,588 \pm 108 \mathrm{~g} / \mathrm{g}$ TSM, respectively. In comparing the 2 - and 24-wk-old airway segments, it will be noted that the mean dose-response relationships to SP were similar. Moreover, at the highest administered dose of SP (i.e., $3 \times 10^{-6} \mathrm{M}$ ), the

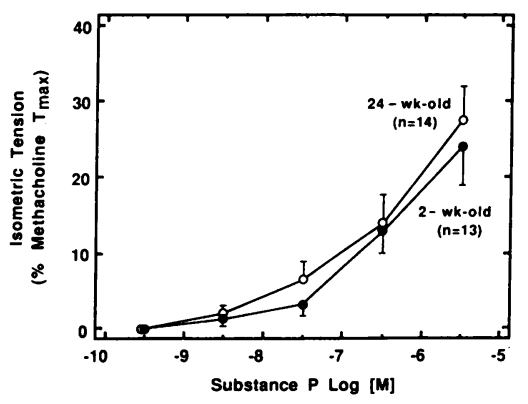

Figure 3. Comparison of mean noncumulative dose-response relationships to SP obtained in 24 wk-old (open circles) and 2-wk-old (solid circles) TSM segments. Isometric tension is expressed as mean \pm SEM percentage of maximal contractile response $\left(T_{\max }\right)$ to methacholine.

mean ( \pm SEM) contractile responses in the 2- and 24-wk-old airway segments amounted to $24( \pm 4.9)$ and $27.7( \pm 4.4) \%$ of the respective $T_{\max }$ responses to methacholine. Thus, in contrast to the above age-dependent differences in SP-induced neuromodulation during electrical field stimulation, there were no maturational differences in the direct contractile effect of SP on the TSM segments.

Effects of ES on SP-induced neuromodulation. To further characterize the pattern of ontogenetic variation in the neuromodulatory influence of SP, for each tissue we determined the SP-induced changes in isometric tension obtained at various levels of electrical field stimulation. Fig. 4 illustrates the agerelated changes in the mean $( \pm$ SEM) isometric tensions to ES obtained in the presence of SP (i.e., $10^{-7} \mathrm{M}$ ), wherein the measurements are expressed as percentages of corresponding baseline responses producing 25,50 , and $100 \%$ of control $T_{\max }$ (i.e., at $\mathrm{ES}_{25}, \mathrm{ES}_{50}$, and $\mathrm{ES}_{100}$, respectively). Here, it will be noted that SP exerted little or no significant neuromodulatory effect at any level of ES before $4 \mathrm{wk}$ of age. During subsequent maturation, on the other hand, potentiation of the contractile responses occurred at all levels of ES, and the latter effect progressively increased with age.

Effect of TTX on ES-induced contractions. Additional experiments were conducted to evaluate whether the above neuromodulatory effect of SP represented a selective neurally mediated action of the neuropeptide over the entire range of electrical field stimulation. In addressing this consideration, the effects of SP were determined before and after inhibition of neural transmission with TTX $\left(4 \times 10^{-6} \mathrm{M}\right)$. As illustrated for a group of 24-wk-old TSM segments in Fig. 5, after the administration of TTX, the contractile responses to ES were virtually abolished in both the absence and presence of $10^{-7} \mathrm{M} \mathrm{SP}$. These data demonstrate that the above SP-induced changes in the contractile responses to ES were dependent on intact neural transmission.

Effect of SP antagonist on SP-induced neuromodulation. We also examined the receptor specificity of the neuromodu-

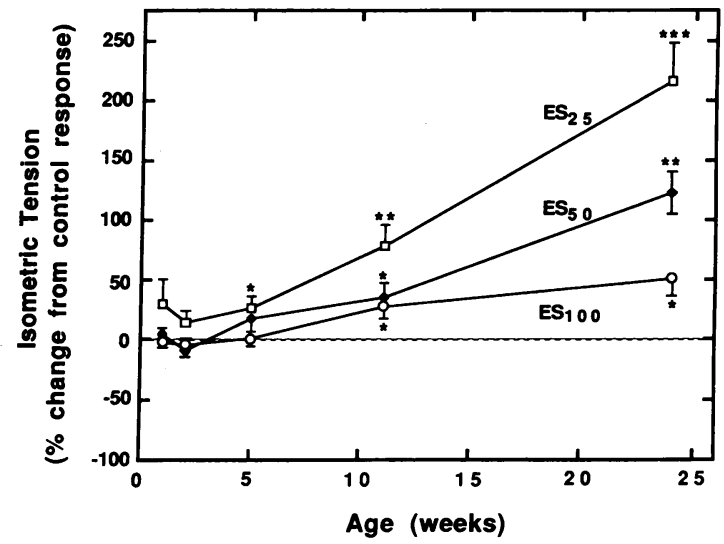

Figure 4. Age-related potentiation of the contractile response to ES induced by $10^{-7} \mathrm{M} \mathrm{SP}$. Data are expressed as mean \pm SEM percentages of the corresponding control contractile responses at 25,50 , and $100 \%$ of peak isometric tension (i.e., $\mathrm{ES}_{25}, \mathrm{ES}_{50}$, and $\mathrm{ES}_{100}$ ). Note that potentiation of ES-induced contractions increases with age, and that the latter age-related effect is more pronounced at lower (i.e., $\mathrm{ES}_{25}$ ) stimulation frequencies. Asterisks denote statistical differences from control contractile responses to ES: ${ }^{*} P<0.05 ;{ }^{* *} P<0.01$; ${ }^{* * *} P<0.005$. 


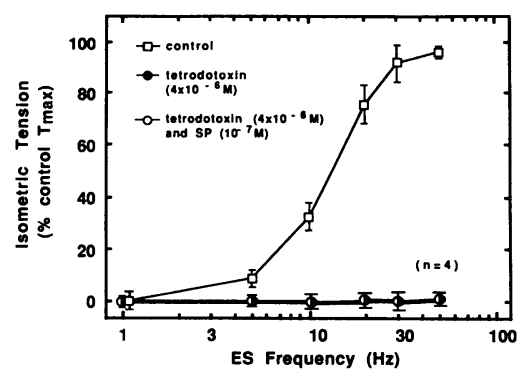

latory effect of SP by evaluating its neuromodulatory action in the absence and presence of the SP antagonist, D-Arg ${ }^{1}$, D-Pro ${ }^{2}$, D-Trp ${ }^{7,9}, \mathrm{Leu}^{11}$-SP. The effects of the SP antagonist are demonstrated in Fig. 6, which compares the mean noncumulative frequency-response relationships obtained in 11-wk-old tissues under initial control conditions of baseline stimulation, in the subsequent presence of $10^{-7} \mathrm{M} \mathrm{SP}$, and thereafter, with the addition of $10^{-5} \mathrm{M}$ of the SP antagonist. As previously noted, after the administration of SP there occurred potentiation of the contractile responses obtained at all levels of ES. On the other hand, $10 \mathrm{~min}$ after the subsequent addition of the SP antagonist, despite the continued presence of SP, the mean frequency-response relationship was shifted back toward the initial control position. Thus, to the extent that the neuromodulatory actions of SP are reversed by a specific SP antagonist, it appears that the enhanced contractile responses to ES in the presence of SP are mediated by its receptor-specific binding.

Since SP has been identified in nerve terminals supplying the airways in both mature and newborn animals (3), we considered the possibility that the lack of any observed neuromodulatory effect in the tracheal segments isolated from young rabbits may be due to the release of endogenous SP established during the course of the initial (i.e., control) ES (4, 8). In this connection, it could be argued that any potential effect of exogenously administered SP may have been masked by the endogenous release of the neuropeptide during ES (4). In addressing this issue we examined the effects of the SP antagonist on the frequency-response relationships obtained in TSM segments isolated from 2-wk-old rabbits. As illustrated in Fig. 7 , the administration of the SP antagonist $\left(10^{-5} \mathrm{M}\right)$ did not appreciably affect the frequency-response relationship to ES. Thus, it appears unlikely that any significant release of SP

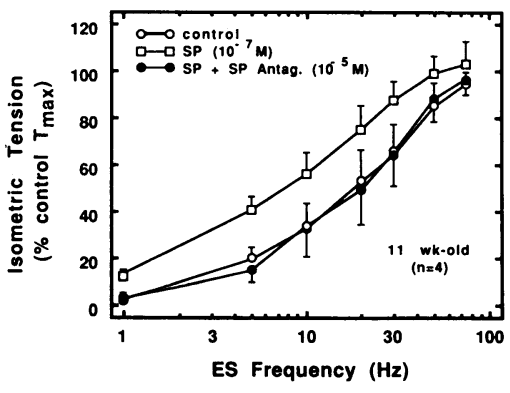

Figure 6. Effect of SP antagonist on ES frequency-response relationships obtained in 11-wk-old airways. Open circles, Baseline frequency-response relationship; open squares, frequency-response curve in the presence of $10^{-7} \mathrm{M}-\mathrm{SP} ;$ solid circles, frequency-response relationship after the administration of an SP antagonist. Data are normalized to control peak tension $\left(T_{\max }\right)$ to ES. Bars, Mean \pm SEM

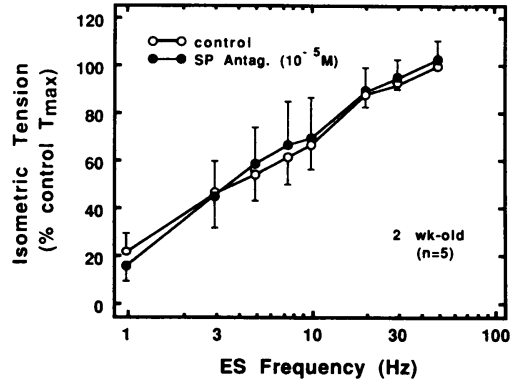

Figure 7. Comparison of mean frequency-response relationships to ES obtained in 2-wk-old TSM segments before (open circles) and after (solid circles) administration of the SP antagonist.

had affected the initial contractile responses to ES in these immature airways.

Interaction of ES sensitivity and age on SP-induced neuromodulation. In a recent study we found that the administration of SP does not influence the underlying cholinergic responsiveness of adult TSM to an administered cholinergic agonist (5). This finding suggested that the augmented contractile response to ES in the presence of SP is attributed to an enhanced presynaptic release of $\mathrm{ACh}$ established during electrical field stimulation (7). In view of the latter, together with the above observations, we questioned whether our observed agedependent changes in the neuromodulatory effect of SP are related to the underlying sensitivity of the tissues to ES. Accordingly, we evaluated the relationship between the degree of SP-induced neuromodulation and their corresponding baseline sensitivity to ES (i.e., ES $_{50}$ values) obtained before SP administration. This relationship is demonstrated for all the airway segments studied in Fig. 8, where the degree of neuromodulation for each tissue is expressed in terms of the percentage change in the magnitude of its contractile response obtained to the same $\mathrm{ES}_{50}$ level of stimulation after the administration of SP. It can be seen that for all the airways studied, the magnitude of SP-induced neuromodulation was closely related to the baseline $\mathrm{ES}_{50}$ values, indicating that the neuromodulatory effect of SP is enhanced in tissues that are intrinsically less sensitive to $\mathrm{ES}$ (i.e., having higher $\mathrm{ES}_{50}$ values). To the extent that this relationship encompasses the data obtained from rabbits of varying age, as well as rabbits of the same age

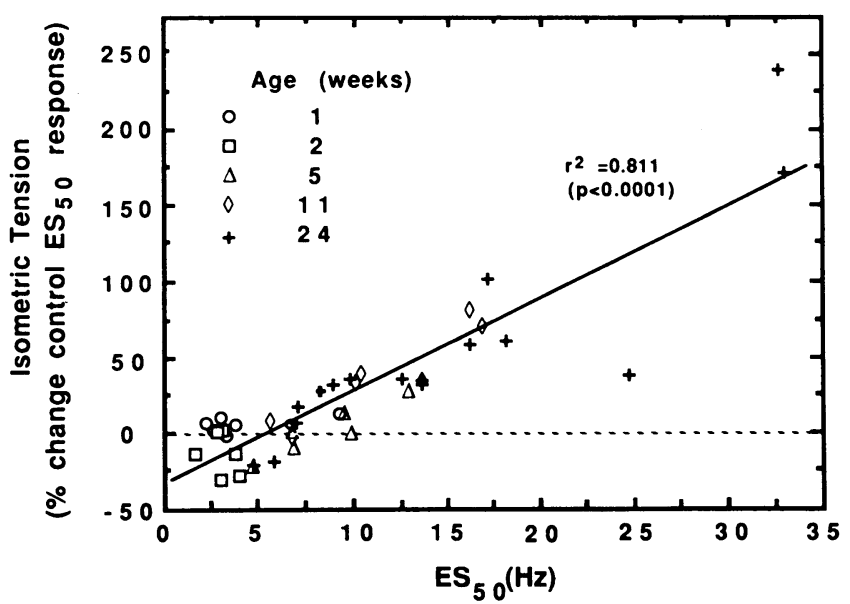

Figure 8. Relationship between the degree of TSM sensitivity to ES (i.e., $\mathrm{ES}_{50}$ values) and the degree of SP-induced neuromodulation from tissues of all ages. Isometric tension (mean \pm SEM) is expressed as percentage of change from control $\mathrm{ES}_{50}$ response. 
depicting considerable variability in their intrinsic sensitivity to ES (e.g., 24-wk-old animals), this finding suggests that the neuromodulatory effect of SP is closely coupled to neural stimulation, rather than to age per se. Indeed, as shown in Table I, after accounting for the interaction of age, the relationship between $\mathrm{ES}_{50}$ and the magnitude of SP-induced neuromodulation remained highly significant. On the other hand, after accounting for the effect the tissue's sensitivity to ES, the age of the TSM segment and the magnitude of SP-induced neuromodulation were no longer significantly related.

\section{Discussion}

SP is a neurokinin that has been localized in unmyelinated fibers innervating the airways in various species including man (1-3). In assessing this physiologic role in regulating airway function, SP has been shown to enhance vascular permeability (9), increase mucous production $(10,11)$, alter epithelial cell ion transport (12), and elicit airway smooth muscle contraction $(2,5,13)$. Collectively, these actions support the notion that SP may play a central role in mediating neurogenic airway inflammation in response to vagal stimulation with electrical $(4,7)$ as well as mechanical and chemical stimuli (14).

In considering its bronchoactive properties, there exists substantial evidence that the airway contractile response to SP is attributed to both its direct action on airway smooth muscle and, at least in both rabbit and ferret airways, its potentiation of $\mathrm{ACh}$ release from cholinergic nerve terminals $(4,6,7)$. The present findings demonstrated that the latter neuromodulatory action of SP is age dependent. In this regard, we found that SP exerts little or no effect on the contractile response to ES of TSM segments isolated from rabbits $<1$ mo of age; however, beyond this age the magnitude of ES-induced contraction is progressively enhanced in the presence of SP. Qualitatively, these observations are in general agreement with our earlier in vivo study, where we found that the bronchoconstrictor response to SP in maturing rabbits includes an atropine-sensitive component that progressively increases during the course of early postnatal development (6). Moreover, in this previous study we also observed that the net in vivo bronchoconstrictor response to SP involving the relatively larger airways (i.e., assessed in terms of changes in respiratory conductance) does

Table I. Interaction of $E S_{50}$ and Age on SP-induced Neuromodulation

\begin{tabular}{llccrr}
\hline \multicolumn{1}{c}{ Source } & Interaction & DF & Sum of squares & F value & PR $>$ F \\
\hline Age & None & 2 & 4,829 & 12.16 & 0.0004 \\
ES $_{50}$ & With age & 1 & 16,128 & 81.20 & 0.0001 \\
ES $_{50}$ & None & 1 & 20,076 & 101.08 & 0.0001 \\
Age & With ES & 2 & 880 & 2.22 & 0.1351 \\
ES $_{50} *$ Age & & 2 & 35 & 0.09 & 0.9158
\end{tabular}

Interaction of age and tissue sensitivity to $\mathrm{ES}$ (i.e., $\mathrm{ES}_{50}$ values) on the magnitude of SP-induced neuromodulation in TSM segments. Magnitude of SP-induced neuromodulation is expressed as percentage change from control $\mathrm{ES}_{50}$ response. Data analysis is based on the SAS general linear models procedure where $D F$ is the degree of freedom, $F$ is the F-test value, and $P R>F$ is the statistical probability (i.e., $P$ value) that the interaction is random. not systematically vary with age. This finding is in further accord with the present in vitro observations demonstrating that the end organ contractile response to SP in TSM does not vary with age. Thus, taken together, our earlier in vivo findings and the present in vitro observations indicate that, whereas the airway neuromodulatory effect of SP is age dependent, the direct contractile response of airway smooth muscle to the neuropeptide (at least involving the larger airway) is not age related.

To elucidate the basis for the observed age-related changes in the neuromodulatory effect of SP, several considerations were addressed. Regarding the tissue selectivity of action of SP, we examined whether the augmented ES-induced contractions in the presence of SP were nonselective to neuronal stimulation and included a direct action on the airway smooth muscle. In addressing this possibility we found that pretreatment with the neurotoxin, TTX, ablated the contractile responses to ES obtained either in the absence or presence of SP. These findings, coupled with earlier evidence that the enhanced ESinduced airway contraction in the presence of SP is cholinergically mediated indicate that the age-dependent differences in the effect of SP on ES-induced contraction were due to an action of the neuropeptide on cholinergic neural elements (i.e. neuromodulation), rather than influencing any nonspecific changes in TSM contraction due to ES per se.

Given the identification of SP-containing nerves in the immature lung (3), we also considered the possibility that endogenous stores of SP may have been released during the initial course of ES-induced airway contraction and, thereby, obscured any potential neuromodulatory action of exogenously administered SP in the younger tissues. In this connection, the recent observation by Sekizawa and associates that an SP antagonist can diminish ES-induced contractions of isolated ferret trachea (4), suggests that endogenous SP may play a neuromodulatory role in these tissues. Accordingly, we examined the effects of an SP antagonist on ES-induced TSM contractions obtained in groups of 2- and 11-wk-old rabbits. We observed that in the presence of the SP antagonist: $(a)$ the neuromodulatory effect of SP was effectively inhibited in older tissues; and $(b)$ the ES-induced contractile responses were unaffected in younger (i.e., 2-wk-old) tissues. These findings respectively demonstrate that the observed neuromodulatory effect of SP in the older tissues was receptor specific, and that the absence of any SP-induced neuromodulation in the younger tissues was probably not related to any prior release of endogenous SP established during the initial course of electrical field stimulation. Moreover, to the extent that in the 11 -wk-old tissues there was virtually no difference between the initial control contractile responses to ES and those obtained in the combined presence of SP and the SP antagonists, our findings further suggest that SP is also not released in these relatively older tissues in amounts sufficient to influence the initial (i.e., control) contractile responses to ES. In this connection, however, it should be emphasized that the above implied absence of endogenous SP release in sufficient quantity to affect TSM contractility to ES in vitro may not accurately reflect the endogenous neuromodulatory contribution of the neuropeptide in vivo or under conditions of altered peptidase activity (4).

In further analyzing the basis for the age-dependent changes in the airway neuromodulatory effect of SP, we found that the magnitude of SP-induced neuromodulation was 
highly correlated to the tissues' intrinsic responsiveness to ES, as reflected by their control $\mathrm{ES}_{50}$ values. Of significance, this close relationship encompassed the data obtained from rabbits of varying postnatal age, as well as those obtained from rabbits of the same age depicting considerable individual variability in their intrinsic sensitivities to ES. In light of this finding, we statistically evaluated the relative interactions of age and the baseline $\mathrm{ES}_{50}$ values with the degree of SP-induced neuromodulation using the SAS general linear model. After accounting for the interaction of age, we found that the relationship between baseline $\mathrm{ES}_{50}$ and the degree of SP-induced neuromodulation remained highly significant. On the other hand, after correcting for the interaction of $\mathrm{ES}_{50}$, the effect of age alone on the magnitude of SP-induced neuromodulation was not statistically significant. Thus, this analysis suggests that our observed age-related change in the neuromodulatory action of SP is attributed to differences in the tissues' intrinsic sensitivities to neural stimulation, rather than their age per se. In general, our younger tissues displayed a relatively greater intrinsic sensitivity to ES (see also reference 15) and, accordingly, these tissues depicted a lesser degree of SP neuromodulation. Of interest, this reciprocal relationship between the degree of SPinduced neuromodulation and intrinsic sensitivity to neural cholinergic stimulation has been previously documented in other neuromodulatory systems. For example, the stimulatory neuromodulatory effect of ACh on dopamine release from nigrostriatal dopaminergic neurons has been shown to be inversely related to the intrinsic level of activity in the dopaminergic nerves (16). In this regard, it has been speculated that in the peripheral and central nervous systems, the dependence of the action of a neuromodulator on the degree of intrinsic activity in the effector neuron may represent a local feedback mechanism that serves to adjust the level of neurotransmitter release under various physiological conditions (17). Notwithstanding the latter, the contribution(s) of other potential mechanisms underlying our observed age-related changes in the airway neuromodulatory action of SP remain to be identified, including maturational differences in the local degradation of the neuropeptide $(4,18)$, the type and density of airway neuronal SP receptors (19), and/or the calcium-dependent ACh release by SP $(20,21)$.

In conclusion, this study was concerned with assessing the maturation of the neuromodulatory action of SP on the contractile response of rabbit TSM to electrical field stimulation and elucidating the age-dependent mechanisms underlying its neuromodulatory effect. Our results demonstrated that: $(a)$ SP does not exert any appreciable neuromodulatory action in TSM isolated from rabbits $<1$ mo old; $(b)$ during subsequent maturation, however, the TSM contractile response to ES becomes progressively potentiated in the presence of SP; $(c)$ the latter augmented contractile response to ES in the presence of SP is selectively dependent on neuronal stimulation, and is attributed to a receptor-specific action of the neuropeptide; and $(d)$ the magnitude of the neuromodulatory effect of SP is closely coupled to the tissues' intrinsic responsivity to neural stimulation, a feature that accounts for the apparent age-related difference in the airway neuromodulatory action of SP.

\section{Acknowledgments}

We thank John LeBreque for his assistance with the statistical analyses and Marcie S. Knight for her technical assistance.

This work was supported in part by National Heart Lung and Blood Institute grant HL-31467.

\section{References}

1. Gamse, R., F. Lembeck, and A. C. Cuello. 1979. Substance P in the vagus nerve: immunochemical and immunohistological evidence for axoplasmic transport. Naunyn-Schmiedebergs Arch. Pharmakol. 306:37-44.

2. Nilsson, G., K. Dahlberg, E. Brodin, E. Sundler, and K. Strandberg. 1977. Distribution and constrictor effect of substance $P$ in guinea pig tracheobronchial tissue. In Substance P. U. S. von Euler and P. Pernow, editors. Raven Press, New York. 75-81.

3. Wharton, J., J. M. Polak, S. R. Bloom, J. A. Will, M. R. Brown, and A. G. E. Pearse. 1979. Substance P-like immunoreactive nerves in the mammalian lung. Invest. \& Cell Pathol. 2:3-19.

4. Sekizawa, K., J. Tamaoki, J. A. Nadel, and D. B. Borson. 1987. Enkephalinase inhibitor potentiates substance P- and electrically induced contraction in ferret trachea. J. Appl. Physiol. 63:1401-1405.

5. Tanaka, D. T., and M. M. Grunstein. 1984. Mechanisms of substance $\mathrm{P}$-induced contraction of rabbit airway smooth muscle. $J$. Appl. Physiol. 57:1551-1557.

6. Grunstein, M. M., D. T. Tanaka, and J. S. Grunstein. 1984. Mechanisms of substance P-induced bronchoconstriction in the maturing rabbit. J. Appl. Physiol. 57:1238-1246.

7. Tanaka, D. T., and M. M. Grunstein. 1986. Effect of substance $P$ on neurally mediated contraction of rabbit airway smooth muscle. $J$. Appl. Physiol. 60:458-463.

8. Leander, S., R. G. G. Grunstrom, and R. Hakanson. 1984. Neuronally mediated noncholinergic contraction of guinea pig bronchial smooth muscle is inhibited by a substance $\mathrm{P}$ antagonist. Agents Actions. 14:3-4.

9. Lundberg, J. M., A. Saria, E. Brodin, S. Rosell, and K. Folkers. 1983. A substance $P$ antagonist inhibits vagally induced increase in vascular permeability and bronchial smooth muscle contraction in the guinea pig. Proc. Natl. Acad. Sci. USA. 80:1120-1124.

10. Baker, A. P., L. M. Hillegrass, D. A. Holden, and W. J. Smith. 1977. Effect of kallidin, substance $P$, and other basic polypeptides on the production of respiratory molecules. Am. Rev. Respir. Dis. 115:811-817.

11. Gashi, A. A., D. B. Borson, W. E. Finkbeiner, J. A. Nadel, and C. B. Basbaum. 1986. Neuropeptides degranulate serous cells of ferret tracheal glands. Am. J. Physiol. 251 (Cell Physiol. 20):C223-C229.

12. Al-Bazzaz, F. J., J. G. Kelsey, and W. D. Kaage. 1985. Substance $P$ stimulation of chloride secretion by canine tracheal mucosa. Am. Rev. Respir. Dis. 131:86-89.

13. Lundberg, J. M., C. R. Martling, and A. Saria. 1983. Substance $P$ and capsaicin-induced contraction of human bronchi. Acta Physiol. Scand. 119:49-53.

14. Lundberg, J. M., and A. Saria. 1983. Capsaicin-induced desensitization of airway mucosa to cigarette smoke, mechanical, and chemical irritants. Nature (Lond.). 302:251-253.

15. Schweiler, G. H., J. S. Douglas, and A. Bouhuys. 1970. Postnatal development of autonomic efferent innervation in the rabbit. Am. J. Physiol. 219:391-397.

16. Giorguieff-Chesselet, M. F., M. L. Kemel, and J. Glowinski. 1979. The presynaptic stimulating effect of acetylcholine on dopamine release is suppressed during activation of nigrostriatal dopaminergic neurons in the cat. Neurosci. Lett. 14:177-182.

17. Chesselet, M. F. 1984. Presynaptic regulation of neurotransmitter release in the brain: fact and hypothesis. Neuroscience. 12:347-375.

18. Shore, S. A., N. P. Stimler-Gerard, S. R. Coats, and J. M. Drazen. 1988. Substance P-induced bronchoconstriction in the guinea pig. Am. Rev. Respir. Dis. 137:331-336.

19. Lee, C. M., L. L. Iversen, M. R. Hanley, and B. E. B. Sandberg. 1982. The possible existence of multiple receptors for substance $P$. Naunyn-Schmiedebergs Arch. Pharmakol. 318:218-287.

20. Donowitz, M., R. Fogel, L. Battisti, and N. Asarkof. 1982. The neurohumoral secretagogues carbachol, substance $\mathbf{P}$ and neurotensin increase $\mathrm{Ca}^{++}$influx and calcium content in rabbit ileum. Life Sci. 31:1929-1937.

21. Yau, W. M., J. A. Dorsett, and M. L. Youther. 1986. Calciumdependent stimulation of acetylcholine release by substance $P$ and vasoactive intestinal peptide. Eur. J. Pharmacol. 120:241-243. 